\begin{tabular}{|c|l|}
\hline Title & Robustness of scale free networks to cascading failures induced by fluctuating loads \\
\hline Author(s) & Mizutaka, Shogo; Y akubo, Kousuke \\
\hline Citation & $\begin{array}{l}\text { Physical review E, 92(1), 12814 } \\
\text { https://doi.org/L0.1103/PhysRevE.92.012814 }\end{array}$ \\
\hline Issue Date & 2015-07-20 \\
\hline Doc URL & http://hdl.handle.net/2115/59769 \\
\hline Rights & ○2015A merican Physical Society \\
\hline Type & article \\
\hline File Information & PhysRevE.92.012814.pdf \\
\hline
\end{tabular}

Instructions for use 


\title{
Robustness of scale-free networks to cascading failures induced by fluctuating loads
}

\author{
Shogo Mizutaka* and Kousuke Yakubo ${ }^{\dagger}$ \\ Department of Applied Physics, Hokkaido University, Sapporo 060-8628, Japan
}

(Received 27 May 2015; published 20 July 2015)

\begin{abstract}
Taking into account the fact that overload failures in real-world functional networks are usually caused by extreme values of temporally fluctuating loads that exceed the allowable range, we study the robustness of scale-free networks against cascading overload failures induced by fluctuating loads. In our model, loads are described by random walkers moving on a network and a node fails when the number of walkers on the node is beyond the node capacity. Our results obtained by using the generating function method show that scale-free networks are more robust against cascading overload failures than Erdős-Rényi random graphs with homogeneous degree distributions. This conclusion is contrary to that predicted by previous works, which neglect the effect of fluctuations of loads.
\end{abstract}

\section{INTRODUCTION}

Modern human societies are supported by various functional networks, such as power grids, the Internet, road systems, and corporate transaction networks [1,2]. Since the function of a network is guaranteed by its global connectivity, a decomposition of the network into disconnected components by failures on network elements (nodes or links) might induce the breakdown of the function, which causes a fatal damage to our daily life. It is thus crucial to elucidate what type of network topology is resilient to failures. In this context, the vulnerability of complex networks against random failures and targeted attacks has been extensively studied [3-8]. In these studies, failed nodes are removed from a network at the same time. In addition to such simultaneous failures, many of actual breakdowns are driven by a chain of failures triggered by initial failures of a single or a few nodes. Initial failures and the subsequent avalanche of failures in functional networks are often induced by loads exceeding node capacities. For instance, a bankruptcy of an insolvent company and subsequent chain bankruptcies due to redistributed debts of the bankrupt company can be regarded as a process of such cascading overload failures in a corporate transaction network.

In order to describe how networks lose their global connectivity by cascading overload failures, Motter and Lai proposed a model in which the load at a node is given by the betweenness centrality of the node [9]. Their model predicts that the initial removal of the highest degree (or highest load) node leads a large-scale cascade and scale-free networks are more fragile against cascading overload failures than homogeneous networks. The vulnerability of scale-free networks has also been found in other models of cascading failures [10-15]. In these models, overload failures are caused by nonfluctuating loads determined uniquely by the network structure that exceed the capacity of each node. It is, however, quite general that the load on a node fluctuates temporally [16-18]. An overload failure takes place when an instantaneous value of fluctuating load exceeds the capacity, as in the cases of river flooding or financial collapse in a country [19]. The property of cascading

\footnotetext{
*s.mizutaka@eng.hokudai.ac.jp

†yakubo@eng.hokudai.ac.jp
}

overload failures induced by extreme events might be largely different from that by nonfluctuating loads.

However, the robustness of networks against cascading failures induced by fluctuating loads has not been widely argued so far [20]. Among several ways to describe fluctuating loads, Kishore et al. modeled them by random walkers on a network $[21,22]$. They calculated the overload probability that the number of random walkers on a node exceeds the predetermined node capacity. Moreover, applying this theory, the network robustness against noncascading overload failures has been studied, in which nodes are simultaneously removed once according to the overload probability [23,24]. Although these theories take into account the temporal fluctuations of loads, cascade processes triggered by the initial overload failures have not been considered. In this paper, we examine the robustness of complex networks against cascading overload failures induced by the extreme value of fluctuating loads. Adopting the random walker model proposed by Kishore et al. and their theory of the overload probability [21,22], we present a simple model to describe cascades of overload failures caused by fluctuating loads and calculate the size of the giant component after completing the cascade by using the generating function formalism. Our main result shows that scale-free networks are more robust against cascading overload failures than homogeneous networks, which is contrary to that predicted by previous works [9-15].

The rest of this paper is organized as follows. In Sec. II we present a model to describe cascading overload failures on a complex network based on the random walker model proposed by Kishore et al. [21,22]. In Sec. III we explain the method to calculate the size of the giant component after completing the cascade process by utilizing the master equation for the probability of a node to have the initial degree $k_{0}$ and the degree $k$ at a cascade step $\tau$ and the generating function formalism. Our results are presented in Sec. IV. Section V is devoted to the summary and concluding remarks.

\section{MODEL}

In a functional network, some sort of flow is often required to realize its functionality and at the same time the flow plays a role of a load in the network, such as electric current in a power grid or packet transfer on the Internet. The relation 
between the average and fluctuation of flow in such networks has been investigated empirically and theoretically [16-18]. These studies elucidated that the flux fluctuations at a node have the same scaling behavior with the fluctuations of the number of random walkers on the node. Inspired by this fact, Kishore et al. modeled fluctuating loads by random walkers moving on a network [21,22], where the number of walkers indicates the amount of loads. The stationary probability to find a random walker on a node of degree $k$ in a connected and undirected network with $M_{0}$ links is given by [25]

$$
p_{k}=\frac{k}{2 M_{0}} \text {. }
$$

Using this relation, the probability $h_{k}(w)$ that $w$ walkers are observed on a node of degree $k$ is presented by

$$
h_{k}(w)=\left(\begin{array}{c}
W_{0} \\
w
\end{array}\right) p_{k}^{w}\left(1-p_{k}\right)^{W_{0}-w},
$$

where $W_{0}$ is the total number of walkers in the network. This leads to a natural definition of the node capacity $q_{k}$ of a node of degree $k$ as

$$
q_{k}=\langle w\rangle_{k}+m \sigma_{k},
$$

where $\langle w\rangle_{k}$ and $\sigma_{k}$ are the average and the standard deviation of the binomial distribution $h_{k}(w)$, which are given by $\langle w\rangle_{k}=$ $W_{0} p_{k}$ and $\sigma_{k}=\sqrt{W_{0} p_{k}\left(1-p_{k}\right)}$, respectively, and $m$ is a real positive parameter that characterizes the node tolerance to load. Since the overload probability $F_{W_{0}}(k)$ of a node of degree $k$ is the probability of $w$ to exceed $q_{k}, F_{W_{0}}(k)$ is given by summing up the distribution function (2) over $w$ larger than $q_{k}$. Thus, we have [21]

$$
\begin{aligned}
F_{W_{0}}(k) & =\sum_{w=\left\lfloor q_{k}\right\rfloor+1}^{W_{0}}\left(\begin{array}{c}
W_{0} \\
w
\end{array}\right) p_{k}^{w}\left(1-p_{k}\right)^{W_{0}-w} \\
& =I_{k / 2 M_{0}}\left(\left\lfloor q_{k}\right\rfloor+1, W_{0}-\left\lfloor q_{k}\right\rfloor\right),
\end{aligned}
$$

where $I_{p}(a, b)$ is the regularized incomplete beta function [26] and $\lfloor x\rfloor$ is the greatest integer not greater than $x$. It is important to pay attention to the fact that the overload probability is a decreasing function of degree $k$ [21].

Based on the above overload probability, we model the cascade process of overload failures as follows.

(i) Prepare an initial connected, uncorrelated, and undirected network $\mathcal{G}_{0}$ with $N_{0}$ nodes and $M_{0}$ links, in which $W_{0}$ random walkers exist, and determine the capacity $q_{k}$ of each node according to Eq. (3).

(ii) At each time step $\tau$, assign $W_{\tau}$ random walkers to the network $\mathcal{G}_{\tau}$ at time $\tau$. The total load $W_{\tau}$ is given by

$$
W_{\tau}=\left(\frac{M_{\tau}}{M_{0}}\right)^{r} W_{0},
$$

where $M_{\tau}$ is the total number of links in the network $\mathcal{G}_{\tau}$ and $r$ is a real positive parameter.

(iii) Calculate the overload probability of every node and remove nodes from $\mathcal{G}_{\tau}$ with this probability.

(iv) Repeat (ii) and (iii) until no node is removed in procedure (iii).

In procedure (ii), the total load $W_{\tau}$ is reduced in accordance with the reduction of the network size. In actual cases of cascading failures, the total load is often reduced to some extent during a cascade process to prevent the breakdown of the network function. For instance, when a problem arises in a power supply system due to a natural disaster, the temporary restriction of electricity use is sometimes introduced to avoid a large-scale breakdown of the power grid, as in the case of the Tohoku earthquake and tsunami in 2011 [27]. In addition, when a company goes bankrupt, a large-scale chain bankruptcy could be prevented by the reduction of the total debt (loads) on the transaction network by means of, for example, a special low-interest lending facilities for companies having business relationships with the bankrupt company or the injection of taxpayers' money. The quantity $W_{\tau}$ given by Eq. (5) represents such a reduction of the total load during the cascade process. The exponent $r$ characterizes how quickly the total load decreases with decreasing the network size, which we call hereafter the load reduction parameter. Although the initial network $\mathcal{G}_{0}$ is connected, the network $\mathcal{G}_{\tau}$ at cascade step $\tau$ is not necessarily connected. For an unconnected network $\mathcal{G}_{\tau}$, we assume that $W_{\tau}$ random walkers are distributed to components in proportion to the numbers of links in these components. Namely, the load allocated to the $\alpha$ th component is given by

$$
W_{\tau}^{\alpha}=\frac{M_{\tau}^{\alpha}}{M_{\tau}} W_{\tau},
$$

where $M_{\tau}^{\alpha}$ is the number of links in the $\alpha$ th component.

In procedure (iii), the overload probability during the cascade process cannot be calculated straightforwardly by Eq. (4). First, the degree $k$ of a node in the network $\mathcal{G}_{\tau}$ at step $\tau$ is not the same as its initial degree $k_{0}$. Since the probability to find a random walker on a node in $\mathcal{G}_{\tau}$ is a function of the present degree $k$ of the node while the node capacity is determined by its initial degree $k_{0}$, the overload probability is presented by Eq. (4) with replacing $q_{k}$ by $q_{k_{0}}$. Moreover, when the network $\mathcal{G}_{\tau}$ is unconnected, the overload probability must be calculated in each component, because random walkers in a component cannot move beyond the component. Therefore, the overload probability of a node of degree $k$, whose initial degree is $k_{0}$, in the $\alpha$ th component is given by

$$
F_{W_{\tau}^{\alpha}}\left(k_{0}, k\right)=I_{k / 2 M_{\tau}^{\alpha}}\left(\left\lfloor q_{k_{0}}\left(W_{0}\right)\right\rfloor+1, W_{\tau}^{\alpha}-\left\lfloor q_{k_{0}}\left(W_{0}\right)\right\rfloor\right),
$$

where $M_{\tau}^{\alpha}$ and $W_{\tau}^{\alpha}$ are again the total number of links and the number of random walkers in the $\alpha$ th component of $\mathcal{G}_{\tau}$, respectively.

The robustness of a network against cascading overload failures described above is evaluated by the relative size of the giant component $S_{\mathrm{f}} \equiv N_{\mathrm{f}} / N_{0}$, where $N_{\mathrm{f}}$ is the number of nodes in the largest component of the network $\mathcal{G}_{\mathrm{f}}$ at the final stage of the cascade process. More specifically, the robustness of the network is measured by the load reduction parameter $r_{\mathrm{c}}$ above which the relative size $S_{\mathrm{f}}$ becomes finite. A network providing a smaller $r_{\mathrm{c}}$ can be regarded to be more robust in the sense that there exists a giant component even if the total load is slowly reduced in accordance with the reduction of the network size during the cascade process.

\section{SIZE OF THE GIANT COMPONENT}

In order to assess the robustness of a network, we calculate the relative size $S_{\tau}$ of the giant component in the network $\mathcal{G}_{\tau}$ at 
cascade step $\tau$. The calculation of $S_{\tau}$ requires information on the load $W_{\tau}^{\alpha}$ to obtain the overload probability $F_{W_{\tau}^{\alpha}}\left(k_{0}, k\right)$ for each component. It is, however, difficult to find an analytical expression of $W_{\tau}^{\alpha}$. Thus, we assume that random walkers can jump to other components with a small probability, which enables us to estimate the stationary probability to find a walker on a node of degree $k$ by

$$
p_{k}=\frac{k}{2 M_{\tau}},
$$

instead of $p_{k}^{\alpha}=k / 2 M_{\tau}^{\alpha}$, and the overload probability by

$$
F_{W_{\tau}}\left(k_{0}, k\right)=I_{k / 2 M_{\tau}}\left(\left\lfloor q_{k_{0}}\left(W_{0}\right)\right\rfloor+1, W_{\tau}-\left\lfloor q_{k_{0}}\left(W_{0}\right)\right\rfloor\right),
$$

instead of Eq. (7). This simplification does not largely change the overload probability if the number of links in a component is large enough. The reason is the following. The probability that $w$ walkers are found on a degree- $k$ node in the $\alpha$ th component containing $W_{\tau}^{\alpha}$ walkers is written as

$$
h\left(w ; W_{\tau}^{\alpha}, p_{k}^{\alpha}\right)=\left(\begin{array}{c}
W_{\tau}^{\alpha} \\
w
\end{array}\right)\left(p_{k}^{\alpha}\right)^{w}\left(1-p_{k}^{\alpha}\right)^{W_{\tau}^{\alpha}-w},
$$

if random walkers are confined in the component. The average and the standard deviation of this binomial distribution function of $w$ are $\langle w\rangle_{k}=W_{\tau}^{\alpha} p_{k}^{\alpha}=W_{\tau} k / 2 M_{\tau}$ and $\sigma_{k}=$ $\sqrt{\langle w\rangle_{k}\left(1-k / 2 M_{\tau}^{\alpha}\right)}$, respectively, where we used Eq. (6) and the relation $p_{k}^{\alpha}=k / 2 M_{\tau}^{\alpha}$. In the case that random walkers are allowed to jump between components, on the other hand, the probability to find $w$ walkers on a degree- $k$ node is given by $h\left(w ; W_{\tau}, p_{k}\right)$. The average $\langle w\rangle_{k}^{\prime}$ of this distribution function coincides with the average $\langle w\rangle_{k}$ of $h\left(w ; W_{\tau}^{\alpha}, p_{k}^{\alpha}\right)$. Although the standard deviation $\sigma_{k}^{\prime}=\sqrt{\langle w\rangle_{k}\left(1-k / 2 M_{\tau}\right)}$ of $h\left(w ; W_{\tau}, p_{k}\right)$ is larger than $\sigma_{k}$ of $h\left(w ; W_{\tau}^{\alpha}, p_{k}^{\alpha}\right), \sigma_{k}^{\prime}$ is not very different from $\sigma_{k}$ if $M_{\tau}^{\alpha}$ (thus $M_{\tau}$ ) is large enough. Therefore, both distribution functions $h\left(w ; W_{\tau}^{\alpha}, p_{k}^{\alpha}\right)$ and $h\left(w ; W_{\tau}, p_{k}\right)$ with the same average and similar widths are close to each other. Because of this similarity, $F_{W_{\tau}^{\alpha}}\left(k_{0}, k\right)=$ $\sum_{w \geqslant\left\lfloor q_{k_{0}}\right\rfloor+1} h\left(w ; W_{\tau}^{\alpha}, p_{k}^{\alpha}\right)$, which leads Eq. (7), can be approximated by $F_{W_{\tau}}\left(k_{0}, k\right)=\sum_{w \geqslant\left\lfloor q_{0}\right\rfloor+1} h\left(w ; W_{\tau}, p_{k}\right)$, giving Eq. (9).

We calculate the relative size $S_{\tau}$ of the giant component by using the generating function method [28]. To this end, here we slightly modify the rule of procedure (ii) described in Sec. II so that overloaded nodes are not removed but are left in the system as zero-degree nodes for which random walkers never visit. This does not influence any results in this work, except that the total number of nodes in $\mathcal{G}_{\tau}$ remains constant at $N_{0}$, which makes the theoretical treatment easier. In spite of this modification, we will continue to use the expression "remove a node" for simplicity, but this actually means "remove all links from a node." The generating function method then enables us to calculate $S_{\tau}$ if the network $\mathcal{G}_{\tau}$ is uncorrelated and the degree distribution function $P_{\tau}(k)$ of $\mathcal{G}_{\tau}$ is given. In order to estimate $P_{\tau}(k)$, we introduce the probability $\Pi_{\tau}\left(k_{0}, k\right)$ that a randomly chosen node has the degree $k$ and the initial degree $k_{0}$. Obviously, the probability $\Pi_{\tau}\left(k_{0}, k\right)$ is related to the degree distribution $P_{\tau}(k)$ through

$$
P_{\tau}(k)=\sum_{k_{0} \geqslant k} \Pi_{\tau}\left(k_{0}, k\right) .
$$

As a special case we have, at $\tau=0$,

$$
\Pi_{0}\left(k_{0}, k\right)=P_{0}(k) \delta_{k_{0} k},
$$

because $k$ is always equal to $k_{0}$ in the initial network $\mathcal{G}_{0}$. To obtain the probability $\Pi_{\tau}\left(k_{0}, k\right)$, we further introduce the probability $\phi_{\tau}(k)$ that the overload failure occurs on a node connected to a node of degree $k$ in the network $\mathcal{G}_{\tau}$. Considering that $\Pi_{\tau}\left(k_{0}, k\right) / P_{\tau}(k)$ represents the probability of a degree- $k$ node chosen randomly from $\mathcal{G}_{\tau}$ to have the initial degree $k_{0}$, the probability $\phi_{\tau}(k)$ is expressed as

$$
\phi_{\tau}(k)=\sum_{k_{0}} \sum_{k^{\prime}=1}^{k_{0}} P_{\tau}\left(k^{\prime} \mid k\right) \frac{\Pi_{\tau}\left(k_{0}, k^{\prime}\right)}{P_{\tau}\left(k^{\prime}\right)} F_{W_{\tau}}\left(k_{0}, k^{\prime}\right),
$$

where $P_{\tau}\left(k^{\prime} \mid k\right)$ is the conditional probability that a node of degree $k$ is connected to a node of degree $k^{\prime}$. Since the network $\mathcal{G}_{0}$ has no degree correlations, $\mathcal{G}_{\tau}$ generated by removing nodes randomly from $\mathcal{G}_{0}$ with a probability depending only on the degree is also uncorrelated [29]. Therefore, the conditional probability is presented by $P_{\tau}\left(k^{\prime} \mid k\right)=k^{\prime} P_{\tau}\left(k^{\prime}\right) /\langle k\rangle_{\tau}$, where $\langle k\rangle_{\tau}$ is the average degree of $\mathcal{G}_{\tau}$, and $\phi_{\tau}$ is written as

$$
\phi_{\tau}=\sum_{k_{0}} \sum_{k^{\prime}=1}^{k_{0}} \frac{k^{\prime} \Pi_{\tau}\left(k_{0}, k^{\prime}\right)}{\langle k\rangle_{\tau}} F_{W_{\tau}}\left(k_{0}, k^{\prime}\right),
$$

which is independent of $k$. The probability $\Pi_{\tau}\left(k_{0}, k\right)$ is equal to the probability that a randomly chosen node from $\mathcal{G}_{\tau-1}$ has the initial degree $k_{0}$ and the degree of this node becomes $k$ during the cascade from $\mathcal{G}_{\tau-1}$ to $\mathcal{G}_{\tau}$. Thus, we can set up the master equation for $\Pi_{\tau}\left(k_{0}, k\right)$ as

$$
\begin{aligned}
\Pi_{\tau}\left(k_{0}, k\right)= & \sum_{k^{\prime} \geqslant k} \Pi_{\tau-1}\left(k_{0}, k^{\prime}\right)\left\{\left(\begin{array}{l}
k^{\prime} \\
k
\end{array}\right) \phi_{\tau-1}^{k^{\prime}-k}\left(1-\phi_{\tau-1}\right)^{k}\right. \\
& \left.\times\left[1-F_{W_{\tau-1}}\left(k_{0}, k^{\prime}\right)\right]+\delta_{k 0} F_{W_{\tau-1}}\left(k_{0}, k^{\prime}\right)\right\} .
\end{aligned}
$$

The right-hand side of this equation represents the probability that a degree- $k^{\prime}$ node in $\mathcal{G}_{\tau-1}$ becomes a node of degree $k$. The first term describes the situation that the degree- $k^{\prime}$ node survives and $k^{\prime}-k$ nodes adjacent to this node are removed by overload failures. The second term stands for the case that the degree- $k^{\prime}$ node itself experiences an overload failure and becomes a zero-degree node. Solving numerically Eq. (15) with the aid of Eq. (14), we can obtain the time evolution of the probability $\Pi_{\tau}\left(k_{0}, k\right)$ and the degree distribution $P_{\tau}(k)$ by Eq. (11). The relative size $S_{\tau}$ of the giant component at cascade step $\tau$ is calculated by [28]

$$
S_{\tau}=1-\sum_{k} P_{\tau}(k) u^{k},
$$

where $u$ is the smallest non-negative solution of the transcendental equation

$$
u=G_{1}^{(\tau)}(u)
$$

with $G_{1}^{(\tau)}(x)$ the generating function of the remaining degree distribution, which is defined by

$$
G_{1}^{(\tau)}(x)=\frac{1}{\langle k\rangle_{\tau}} \sum_{k}(k+1) P_{\tau}(k+1) x^{k} .
$$




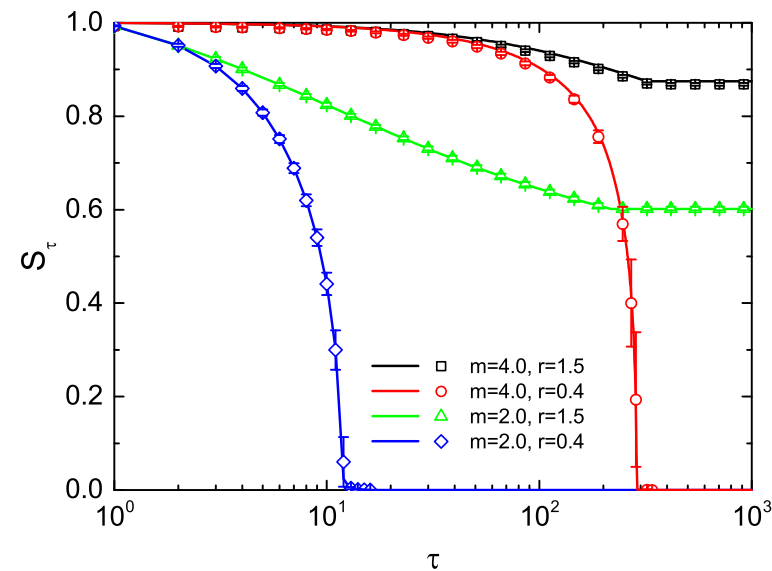

FIG. 1. (Color online) Relative size $S_{\tau}$ of the giant component as a function of the cascade step $\tau$. The initial network is an Erdős-Rényi random graph with 5000 nodes and 12500 links, which implies that the initial average degree is $\langle k\rangle_{0}=5.0$. The symbols indicate the results obtained by numerical simulations following the cascade process from (i) to (iv) described in Sec. II and averaging over 50 network realizations. Error bars represent one standard deviation from the mean values. The lines show the results calculated by the analytical method explained in Sec. III. The total number of walkers at the initial cascade step is $W_{0}=25000$. The values of the node tolerance parameter $m$ and the load reduction parameter $r$ are displayed in the figure.

According to procedure (iv) stated in Sec. II, the cascade process must be terminated when no node experiences overload failures. In an actual calculation, we stop the cascade process at the step $\tau$ satisfying the condition

$$
\sum_{k, k_{0}} F_{W_{\tau}}\left(k_{0}, k\right) \Pi_{\tau}\left(k_{0}, k\right)<\frac{1}{N_{0}} .
$$

This condition implies that the expectation number of overloaded nodes becomes less than unity.

It should be noted that the above formalism is based on the approximation that random walkers are allowed to jump over components with a small probability. In order to evaluate the accuracy of this approximation, we compare the time evolutions of $S_{\tau}$ for an Erdôs-Rényi random graph calculated by both the method explained in this section and numerical simulations following the cascade process from (i) to (iv) described in Sec. II. The results shown in Fig. 1 indicate that $S_{\tau}$ calculated by Eq. (16) agrees quite well with the simulation result, which reflects the high accuracy of the approximation.

\section{RESULTS}

We assess the robustness of networks against cascading overload failures by computing the relative size $S_{\mathrm{f}}$ of the giant component at the final stage of the cascade process as a function of the load reduction parameter $r$. In this work, we examined two types of initial networks, namely, homogeneous Erdős-Rényi (ER) random graphs and scale-free (SF) networks with inhomogeneous degree distributions. An SF network is generated by the configuration model [30] with the degree

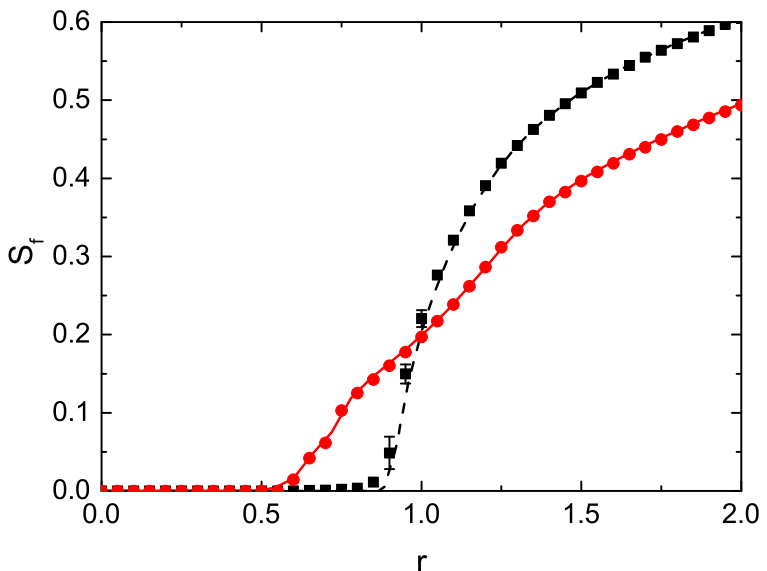

FIG. 2. (Color online) Relative size $S_{\mathrm{f}}$ of the giant component at the final stage of the cascade process as a function of the load reduction parameter $r$, for an ER random graph (black dashed line and solid squares) and an SF network (red solid line and solid circles). The SF network has the degree distribution given by Eq. (20) with $\gamma=2.5$. The lines represent the results calculated by the analytical method explained in Sec. III. The symbols indicate the simulation results obtained by following numerically the cascade process from (i) to (iv) described in Sec. II and averaging over 50 samples. Only error bars larger than the symbol size are shown. In this calculation, we set $N_{0}=10^{4}, M_{0}=2 \times 10^{4}, m=2.0$, and $W_{0}=2 M_{0}$.

distribution given by

$$
P_{0}(k)= \begin{cases}\frac{C}{k^{\gamma}+d^{\gamma}} & \text { for } k \geqslant k_{\min } \\ 0 & \text { for } k<k_{\min },\end{cases}
$$

where $d$ is a real positive parameter and $\gamma$ is the exponent describing the asymptotic power-law form of the degree distribution, i.e., $P_{0}(k) \propto k^{-\gamma}$ for $k \gg d$. In Eq. (20), $k_{\min }$ is the minimum degree and $C$ is the normalization constant. The parameters $d$ and $k_{\min }$ can control the average degree $\langle k\rangle_{0}$ for a fixed value of $\gamma$. Throughout this paper the minimum degree is fixed at $k_{\min }=2$. Figure 2 shows the $r$ dependence of $S_{\mathrm{f}}$ for an ER random graph (black dashed line and solid squares) and for an SF network (red solid line and solid circles). Both networks have $N_{0}=10^{4}$ nodes and $M_{0}=2 \times 10^{4}$ links, which implies that the initial average degree is $\langle k\rangle_{0}=4.0$. The initial total load is chosen as $W_{0}=2 M_{0}$ and the node capacity $q_{k}$ is determined by Eq. (3) with $m=2.0$. For the SF network, the exponent $\gamma$ is set as $\gamma=2.5$ and $d$ is tuned to satisfy $\langle k\rangle_{0}=4.0$. The solid and dashed lines in Fig. 2 indicate the results calculated by the analytical method explained in Sec. III, whereas the symbols represent $S_{\mathrm{f}}$ obtained by simulating numerically the cascade process from (i) to (iv) described in Sec. II. For both types of networks, the analytical results agree quite well with the numerical ones. As shown in Fig. 2, there exists a value $r_{\mathrm{c}}\left(N_{0}\right)$ below which $S_{\mathrm{f}}=0$ and above which $S_{\mathrm{f}}>0$. This implies that a global cascade of overload failures occurs if the total load $W_{\tau}$ is reduced during the cascade more slowly than the reduction scheme (5) with $r=r_{\mathrm{c}}\left(N_{0}\right)$, while a finite fraction of nodes survives the cascade if the reduction of $W_{\tau}$ is faster. The fact that the value of $r_{\mathrm{c}}\left(N_{0}\right)$ for the SF network is smaller than that for the ER random graph suggests that SF networks are more 


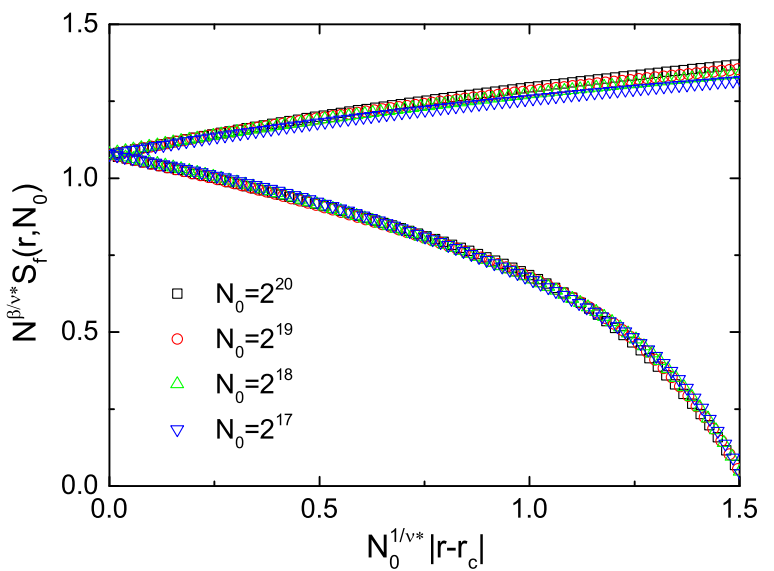

FIG. 3. (Color online) Scaling plot of $S_{\mathrm{f}}\left(r, N_{0}\right)$ for ER random graphs of several sizes $\left(N_{0}=2^{17}, 2^{18}, 2^{19}\right.$, and $\left.2^{20}\right)$. The average degree of these networks is set to be $\langle k\rangle_{0}=4$.0. The calculations have been done under the condition of $W_{0}=N_{0}\langle k\rangle_{0}$ and $m=2.0$. In this plot, we choose $r_{\mathrm{c}}=1.84, \beta=1.27$, and $1 / v_{r}^{*}=19.45$. Two branches correspond to the percolating and nonpercolating phases.

robust to cascading overload failures than ER random graphs. This tendency is opposite to what was predicted by previous works [9-15].

The above conclusion, however, is obtained for finite-size networks. It is crucial to study the robustness of infinitely large SF networks against cascading overload failures. If the change of $S_{\mathrm{f}}$ at $r=r_{\mathrm{c}}\left(N_{0}\right)$ provides a critical transition when $N_{0}$ goes to infinity, we can use the finite-size scaling analysis to calculate $r_{\mathrm{c}}$ in the thermodynamic limit. According to the finite-size scaling theory, the relative size of the giant component in the network $\mathcal{G}_{\mathrm{f}}$ at the final stage of the cascade process starting from the initial network $\mathcal{G}_{0}$ of size $N_{0}$ is expressed as

$$
S_{\mathrm{f}}\left(r, N_{0}\right)=N_{0}^{-\beta / v^{*}} \Phi\left(N_{0}^{1 / v^{*}}\left|r-r_{\mathrm{c}}\right|\right),
$$

where the correlation volume exponent $v^{*}$ characterizes the divergence of the number of nodes $N_{\xi}$ within the correlation volume as $N_{\xi} \propto\left|r-r_{\mathrm{c}}\right|^{-v^{*}}$ in the infinite system, $\beta$ is the critical exponent for the approach to zero of $S_{\mathrm{f}}(r, \infty), r_{\mathrm{c}}$ is the critical load reduction parameter in the thermodynamic limit, and $\Phi(x)$ is a scaling function. Therefore, if the suitable values of the parameters $v^{*}, \beta$, and $r_{\mathrm{c}}$ are selected, the quantity $N_{0}^{\beta / \nu^{*}} S_{\mathrm{f}}\left(r, N_{0}\right)$ as a function of $N_{0}^{1 / v^{*}}\left|r-r_{\mathrm{c}}\right|$ collapses onto a single curve for various values of $r$ and $N_{0}$. Figure 3 shows such a plot for ER random graphs of different sizes with the use of the $r$ dependence of $S_{\mathrm{f}}\left(r, N_{0}\right)$ calculated by the method explained in Sec. III and the best-fit values of $v^{*}, \beta$, and $r_{\mathrm{c}}$. The fact that all data collapse onto a single curve implies that the transition at $r=r_{\mathrm{c}}$ can be considered as a critical phenomenon. Similar scaling behaviors have been confirmed for SF networks.

The criticality of the network $\mathcal{G}_{\mathrm{f}}$ at $r=r_{\mathrm{c}}$ has also been confirmed by the fractal property of the giant component in $\mathcal{G}_{\mathrm{f}}$. The fractality of complex networks is widely investigated by the box covering algorithm [31-37]. If the minimum number $N_{\mathrm{B}}\left(l_{\mathrm{B}}\right)$ of subgraphs of radius $l_{\mathrm{B}}$ required to cover a given

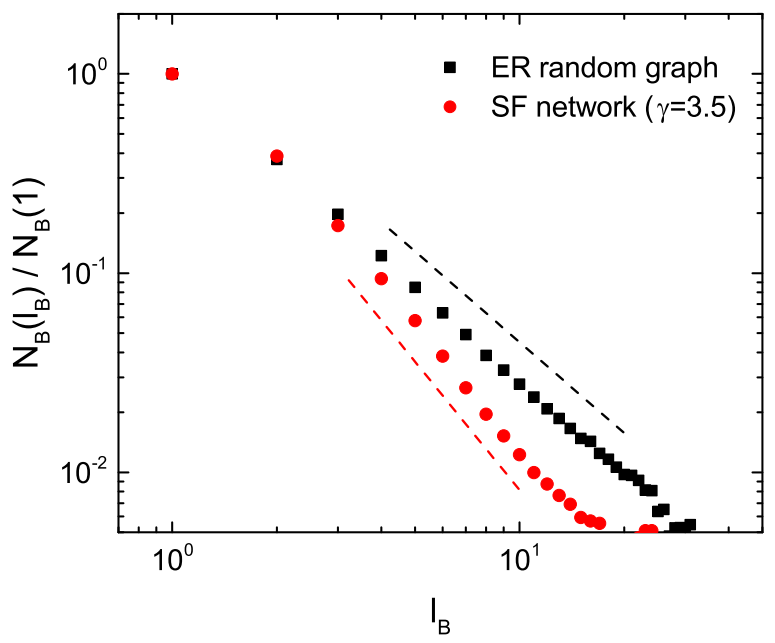

FIG. 4. (Color online) Number of subgraphs $N_{\mathrm{B}}\left(l_{\mathrm{B}}\right)$ for the giant components in $\mathcal{G}_{\mathrm{f}}$ obtained numerically by simulating the cascade process with $r=r_{\mathrm{c}}\left(N_{0}\right)$ starting from an ER random graph (black solid squares) and an SF network with $\gamma=3.5$ (red solid circles). The number of nodes and the average degree of both of the initial networks are $N_{0}=10^{4}$ and $\langle k\rangle_{0}=4.0$, respectively. The calculations have been done under the condition of $W_{0}=N_{0}\langle k\rangle_{0}$ and $m=2.0$. The results are averaged over 100 samples. The longitudinal axis is rescaled by $N_{\mathrm{B}}\left(l_{\mathrm{B}}\right)$ at $l_{\mathrm{B}}=1$. The dashed lines are merely guides to the eye.

connected network satisfies the relation

$$
N_{\mathrm{B}}\left(l_{\mathrm{B}}\right) \propto l_{\mathrm{B}}^{-d_{\mathrm{B}}},
$$

the network is considered to be fractal with the fractal dimension $d_{\mathrm{B}}$ [31]. We calculated, by using the compact-boxburning algorithm [38], $N_{\mathrm{B}}\left(l_{\mathrm{B}}\right)$ for giant components included in networks $\mathcal{G}_{\mathrm{f}}$ at the final stage of the cascade process with $r=r_{\mathrm{c}}\left(N_{0}\right)$ starting from both an ER random graph and an SF network. The giant components are obtained numerically by simulating the cascade process described in Sec. II. The results shown in Fig. 4 indicate that the structures of these giant components exhibit the fractal nature, which supports the criticality of $\mathcal{G}_{\mathrm{f}}$ at $r=r_{\mathrm{c}}$. The fractal dimension for the ER random graph is $d_{\mathrm{B}}=1.54 \pm 0.01$, while $d_{\mathrm{B}}=2.13 \pm 0.02$ for the SF network with $\gamma=3.5$. These values of $d_{\mathrm{B}}$ are different from those of giant components at the critical point of the conventional percolation with random node removals, which are $d_{\mathrm{B}}=2$ for ER random graphs (or SF networks with $\gamma \geqslant 4)$ and $d_{\mathrm{B}}=(\gamma-2) /(\gamma-3)$ for SF networks with $3<\gamma<4$ [39]. Such a discrepancy is, of course, due to the difference in ways of node removals. In the cascade process, nodes that will be removed at the cascade step $\tau$ depend strongly on nodes removed at $\tau-1$, as in the case of a fire spread for which a portion remaining unburned has a different structure from that of a survival from random removals.

The critical load reduction parameter $r_{\mathrm{c}}$ calculated by the finite-size scaling method is plotted in Fig. 5 as a function of the exponent $\gamma$ of SF networks. In this figure, $r_{\mathrm{c}}$ for the ER random graph is also indicated by the horizontal dashed line. The results only for $\gamma \geqslant 5$ are presented here, because for a small value of $\gamma$ the computation of $\Pi_{\tau}\left(k_{0}, k\right)$ requires 


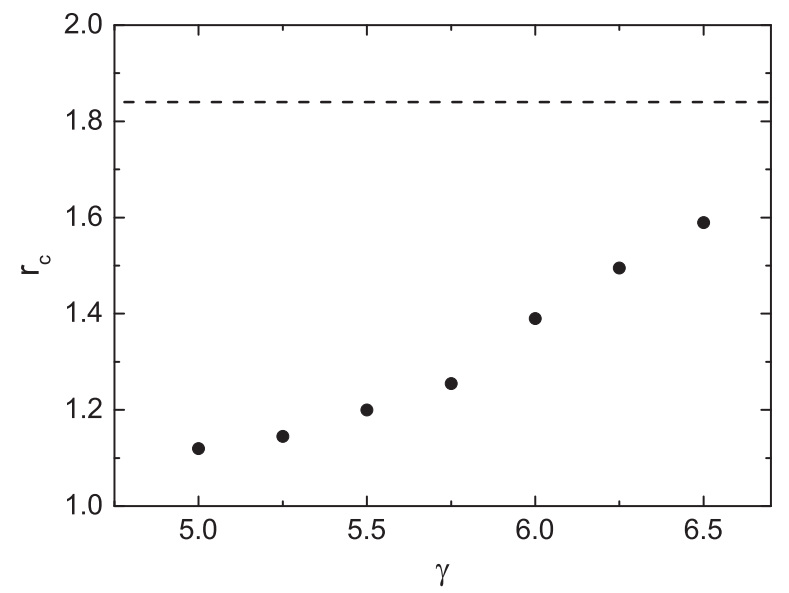

FIG. 5. Critical load reduction parameter $r_{\mathrm{c}}$ as a function of the exponent $\gamma$ in the thermodynamic limit. In this calculation for SF networks, we tune the parameter $d$ in Eq. (20) to satisfy $\langle k\rangle_{0}=4.0$ for various values of $\gamma$. The values of $r_{\mathrm{c}}$ are estimated through the finite-size scaling analysis for $S_{\mathrm{f}}\left(r, N_{0}\right)$ calculated by the method described in Sec. III under the condition of $W_{0}=\langle k\rangle_{0} N_{0}$ and $m=$ 2.0. The horizontal dashed line is the result for the ER random graph $(\gamma \rightarrow \infty)$ with $\langle k\rangle_{0}=4.0$.

a long CPU time due to the increase of the maximum degree $k_{\max }$ of the initial network $\mathcal{G}_{0}$ associated with a decrease of $\gamma$ as is given by $k_{\max } \propto N_{0}^{1 / \gamma}$. We see from Fig. 5 that $r_{\mathrm{c}}$ is an increasing function of $\gamma$. This implies that the enhancement of the SF property by decreasing $\gamma$ makes networks robust to cascading overload failures even in the thermodynamic limit. It should be emphasized that $r_{\mathrm{c}}$ is always positive for any $\gamma$. This is because in any network a cascade of overload failures under $r=0$ never stops until the network collapses completely. Our result, which is contrary to previous predictions [9-15], comes from the fact that the overload probability is a decreasing function of degree $k$. At the first step of the cascade process, nodes with small degrees are more likely to be removed according to the overload probability $F_{W_{0}}(k)$. A low-degree node tends to be connected to a node with large degree in an uncorrelated SF network. The overload probability of a high-degree node adjacent to the low-degree node that was removed at the first step becomes smaller at the second cascade step than its initial overload probability. Therefore, nodes with relatively small degrees are again preferentially removed also at the second step and so on. It is obvious that SF networks are robust against preferential removals of low-degree nodes [40].

Our model differs from previous models of cascading overload failures in two points. One is that overload failures in our model occur when fluctuating loads exceed the capacities predetermined for nodes, while failures are caused by nonfluctuating loads (or average values of fluctuating loads) exceeding the node capacities in previous models [9-15]. The other difference is in the dynamics of loads on a network. In many of previous works [9-15], the load is defined by flow passing through the shortest path between a pair of nodes. This type of load describes, for example, a packet flow in the Internet and a traffic flow in a road system. On the other hand, loads in our model move randomly on a network, as in the

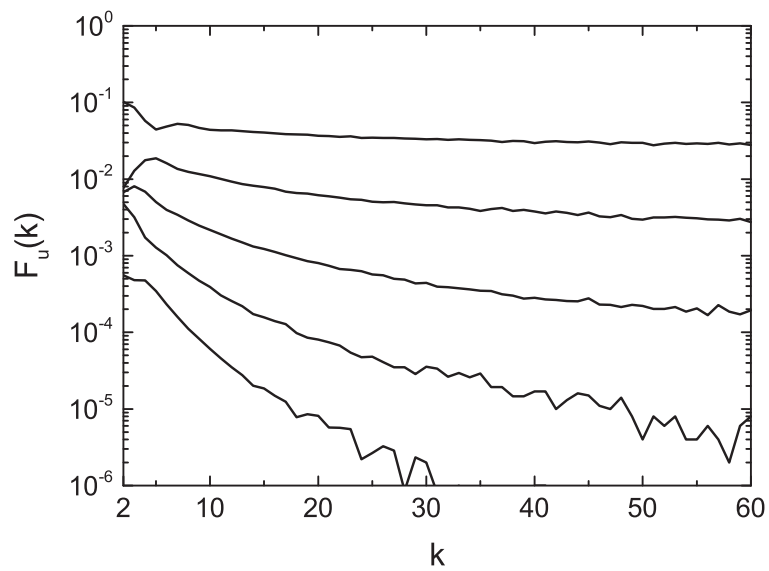

FIG. 6. Degree dependence of the overload probability $F_{u}(k)$ for several values of the node tolerance parameter $m$. The lines from top to bottom represent the results for $m=2.0,3.0,4.0,5.0$, and 6.0 , respectively. The size of the set $V_{u}$ is fixed at $u=500$. The load distribution $\tilde{h}_{k}(w)$ required to calculate $F_{u}(k)$ is numerically evaluated by computing the partial betweenness centrality for each of $10^{4}$ different sets $V_{u}$ selected from each of 30 SF networks with $10^{4}$ nodes. The degree distribution of these SF networks is given by Eq. (20) with $\gamma=2.5$ and $d=0.152$ providing $\langle k\rangle=4.0$.

case of a flow of debt in a corporate transaction network. It is important to clarify whether the robustness of SF networks is caused by the fluctuation of the load or by its random walk behavior.

In order to identify the origin of the robustness, namely, the origin of $F_{W_{0}}(k)$ being a decreasing function of degree $k$, we calculate the overload probability for fluctuating loads imposed by a shortest-path flow. Fluctuating loads carried by flow along the shortest paths have been argued in Ref. [16] to explain the relation between the average flux and the fluctuations. According to this work, we newly define the load $w_{i}$ of the node $i$ by

$$
w_{i}=\sum_{\substack{\left.\left(j, j^{\prime}\right) \in V_{u} \\ j \neq j^{\prime}\right)}} \frac{\sigma_{j j^{\prime}}(i)}{\sigma_{j j^{\prime}}},
$$

where $V_{u}$ is a set of $u$ node pairs that are randomly selected from $\left(N_{0}-1\right)\left(N_{0}-2\right) / 2$ node pairs in $\mathcal{G}_{0}$ excluding the node $i, \sigma_{j j^{\prime}}$ is the total number of shortest paths between the pair $\left(j, j^{\prime}\right)$ in $V_{u}$, and $\sigma_{j j^{\prime}}(i)$ is the number of those paths that pass through $i$. Since the quantity represented by the right-hand side of Eq. (23) is equivalent to the betweenness centrality $b_{i}$ if $V_{u}$ is chosen as the set of all node pairs, namely, $u=\left(N_{0}-1\right)\left(N_{0}-2\right) / 2$, we call the above quantity $w_{i}$ the partial betweenness centrality. The partial betweenness centrality of a node depends on which node pairs are selected for the set $V_{u}$ and thus the load $w_{i}$ fluctuates in accordance with the choice of $V_{u}$. For a large number of different sets $V_{u}$ with $1 \ll u \ll\left(N_{0}-1\right)\left(N_{0}-2\right) / 2$, the distribution $\tilde{h}_{i}(w)$ of the load can be calculated for each node. As the conventional betweenness centrality $b_{i}$ is strongly correlated to the degree $k_{i}$ in an uncorrelated network $[41,42]$, the partial betweenness centrality of a node is also expected to have a correlation with the degree of the node. In fact, we have confirmed numerically that two distribution functions $\tilde{h}_{i}(w)$ and $\tilde{h}_{j}(w)$ of the load 
TABLE I. Robustness and fragility of SF networks against cascading overload failures induced by several types of loads. The fragility for the shortest path flow without fluctuation is a consequence of previous work [9].

\begin{tabular}{lcc}
\hline \hline Load flow & Without fluctuation & With fluctuation \\
\hline shortest path & fragile & robust \\
random walk & fragile & robust \\
\hline \hline
\end{tabular}

on the nodes $i$ and $j$ that have the same degree are close to each other. Therefore, we can define the degree-dependent load distribution $\tilde{h}_{k}(w)$ for the partial betweenness centrality model, which corresponds to the load distribution $h_{k}(w)$ given by Eq. (2) for the random walker model. The node capacity $q_{k}$ is also defined by Eq. (3) with the average load $\langle w\rangle_{k}$ and the standard deviation $\sigma_{k}$ calculated by the distribution function $\tilde{h}_{k}(w)$. Finally, as in the case of the random walker model, the overload probability is defined as the probability that the load of a node of degree $k$ exceeds its capacity $q_{k}$, which is calculated by $\int_{q_{k}}^{\infty} \tilde{h}_{k}(w) d w$. Since $\tilde{h}_{k}(w)$ depends on $u$, we denote this overload probability by $F_{u}(k)$ instead of $F_{W_{0}}(k)$.

Figure 6 shows the degree dependence of $F_{u}(k)$ for several values of the node tolerance parameter $m$. We see that the overload probability $F_{u}(k)$ is a decreasing function of $k$ for any $m$. This implies that SF networks are robust also against cascading overload failures induced by fluctuating shortestpath flow. It is not surprising that the degree dependences of $F_{W_{0}}(k)$ and $F_{u}(k)$ show a similar tendency, because it has been reported that the couplings between the fluctuations and the average of the number of random walkers and the partial betweenness centrality on individual nodes obey the same scaling law [16]. We can also consider a situation that cascading failures are caused by nonfluctuating loads by random walkers. In this case, an overload failure occurs when the average number of walkers $\langle w\rangle_{k}=W_{0} k / 2 M_{0}$ on a node of degree $k$ exceeds the node capacity depending on $\langle w\rangle_{k}$, where $W_{0}$ is the total number of walkers and $M_{0}$ is the number of links in the initial network. Since $\langle w\rangle_{k}$ is proportional to $k$ and the degree of a node correlates closely with the betweenness centrality of the node [41,42], the property of cascading failures induced by nonfluctuating random walking loads is essentially the same as that by loads of the betweenness centrality [9]. Therefore, SF networks are fragile to such cascading failures. All the above arguments can be summarized as shown in Table I. From this table we can conclude that the robustness of SF networks in our model is a consequence of the property that failures are caused by extreme values of fluctuating loads, but not concerned with the random walk behavior of loads.

\section{CONCLUSION}

We have studied the robustness of scale-free (SF) networks against cascading overload failures induced by extreme values of fluctuating loads that exceed the node capacities. In our model, temporally fluctuating loads are treated as random walkers on a network, for which the stationary overload probability has been studied by Kishore et al. [21]. At the first stage of the cascade, nodes are removed from the initial network with this overload probability and the redistribution of loads in the damaged network causes iteratively subsequent failures according to the updated overload probability until no node is expected to be removed. During the cascade process, the total load is reduced in response to the decrease of the number of links in the network under the cascade. How quickly the total load is reduced is characterized by the load reduction parameter $r$. The robustness of a network is measured by the critical load reduction parameter $r_{\mathrm{c}}$ above which the relative size $S_{\mathrm{f}}$ of the giant component at the final cascade stage is finite. We present a formulation to calculate $S_{\mathrm{f}}$ by using the master equation for the probability $\Pi_{\tau}\left(k_{0}, k\right)$ of a node in the network $\mathcal{G}_{\tau}$ at cascade step $\tau$ to have the present degree $k$ and the initial degree $k_{0}$ and by applying the generating function method. Our results for SF networks with degree distribution $P_{0}(k) \sim k^{-\gamma}$ at large $k$ show that $r_{\mathrm{c}}$ increases with the exponent $\gamma$, which implies that SF networks are robust against cascading overload failures in our model as opposed to previous works [9-15]. The robustness of SF networks is explained by the property of the overload probability of being a decreasing function of the degree, which is a consequence of the load fluctuations but not concerned with the random walk behavior of loads.

In our model, the total load does not fluctuate throughout the cascade process though the local load on a node fluctuates. If the total load also fluctuates temporally and the time scale of the fluctuation is faster than that of the cascade process, the overload probability must be different from Eq. (4) or (9). However, we can expect that the overload probability remains a decreasing function of the degree if the magnitude of the total load fluctuation $\Delta W$ is much smaller than the average total load $\langle W\rangle$, which guarantees the robustness of SF networks to cascading overload failures. This is because the standard deviation $\sigma_{k}$ of the fluctuating load $w$ on a node of degree $k$ is proportional to the square root of the average load $\langle w\rangle_{k}$ if $\Delta W \ll\langle W\rangle$, as in the case of the present work. It has been reported, however, that $\sigma_{k}$ becomes proportional to $\langle w\rangle_{k}$ when $\Delta W /\langle W\rangle$ approaches 1 [16]. The change in the property of the load fluctuations may alter drastically the degree dependence of the overload probability and hence the robustness of networks. It is thus important to study how robust SF networks are against cascading failures under large fluctuations of the total load. Furthermore, although we present the formulation to calculate the relative size of the giant component $S_{\mathrm{f}}$ in this work, an analytical expression for the critical load reduction parameter $r_{\mathrm{c}}$ is not found. In addition to solving this problem, the identification of the universality class of the present model is also a subject for future work.

\section{ACKNOWLEDGMENTS}

This work was supported by a Grant-in-Aid for Scientific Research (No. 25390113 and No. 14J01323) from the Japan Society for the Promotion of Science. Numerical calculations in this work were performed in part on the facilities of the Supercomputer Center, Institute for Solid State Physics, University of Tokyo. 
[1] D. Król, D. Fay, and B. Gabryś, Propagation Phenomena in Real World Networks (Springer, Heidelberg, 2015).

[2] L. da F. Costa, O. N. Oliveira, Jr., G. Travieso, F. A. Rodrigues, P. R. V. Boas, L. Antiqueira, M. P. Viana, and L. E. C. da Rocha, Adv. Phys. 60, 329 (2011).

[3] R. Cohen, K. Erez, D. ben-Avraham, and S. Havlin, Phys. Rev. Lett. 85, 4626 (2000).

[4] R. Cohen, K. Erez, D. ben-Avraham, and S. Havlin, Phys. Rev. Lett. 86, 3682 (2001).

[5] M. E. J. Newman, Phys. Rev. Lett. 89, 208701 (2002).

[6] P. Holme, B. J. Kim, C. N. Yoon, and S. K. Han, Phys. Rev. E 65, 056109 (2002).

[7] S. Boccaletti, V. Latora, Y. Moreno, M. Chavez, and D.-U. Hwang, Phys. Rep. 424, 175 (2006).

[8] S. N. Dorogovtsev, A. V. Goltsev, and J. F. F. Mendes, Rev. Mod. Phys. 80, 1275 (2008).

[9] A. E. Motter and Y.-C. Lai, Phys. Rev. E 66, 065102(R) (2002).

[10] P. Holme, Phys. Rev. E 66, 036119 (2002).

[11] P. Crucitti, V. Latora, and M. Marchiori, Phys. Rev. E 69, 045104(R) (2004).

[12] J. J. Wu, H. J. Sun, and Z. Y. Gao, Physica A 386, 407 (2007).

[13] Z. J. Bao, Y. J. Cao, L. J. Ding, and G. Z. Wang, Physica A 388, 4491 (2009).

[14] Y. Xia and D. J. Hill, Physica A 389, 1281 (2010).

[15] B.-L. Dou, X.-G. Wang, and S.-Y. Zhang, Physica A 389, 2310 (2010).

[16] M. A. de Menezes and A.-L. Barabási, Phys. Rev. Lett. 92, 028701 (2004).

[17] S. Meloni, J. Gómez-Gardeñes, V. Latora, and Y. Moreno, Phys. Rev. Lett. 100, 208701 (2008).

[18] Z. Zhou, Z.-G. Huang, L. Huang, Y.-C. Lai, L. Yang, and D.-S. Xue, Phys. Rev. E 87, 012808 (2013).

[19] P. Embrechts, C. Klueppelberg, and T. Mikosch, Modeling Extremal Events: For Insurance and Finance (Springer, Berlin, 1997).
[20] Fluctuation-induced cascading failures have been discussed in D. Heide, M. Schäfer, and M. Greiner, Phys. Rev. E 77, 056103 (2008).

[21] V. Kishore, M. S. Santhanam, and R. E. Amritkar, Phys. Rev. Lett. 106, 188701 (2011).

[22] V. Kishore, M. S. Santhanam, and R. E. Amritkar, Phys. Rev. E 85, 056120 (2012).

[23] S. Mizutaka and K. Yakubo, Phys. Rev. E 88, 012803 (2013).

[24] S. Mizutaka and K. Yakubo, J. Complex Networks 2, 413 (2014).

[25] J. D. Noh and H. Rieger, Phys. Rev. Lett. 92, 118701 (2004).

[26] M. Abramowitz and I. A. Stegun, Handbook of Mathematical Functions (Dover, New York, 1964).

[27] H. Arikawa, Y. Cao, and S. Matsumoto, Energy Res. Soc. Sci. 2, 12 (2014).

[28] M. E. J. Newman, S. H. Strogatz, and D. J. Watts, Phys. Rev. E 64, 026118 (2001).

[29] A. Srivastava, B. Mitra, N. Ganguly, and F. Peruani, Phys. Rev. E 86, 036106 (2012).

[30] M. Molloy and B. Reed, Random Struct. Algor. 6, 161 (1995).

[31] C. Song, S. Havlin, and H. A. Makse, Nature (London) 433, 392 (2005).

[32] C. Song, S. Havlin, and H. A. Makse, Nat. Phys. 2, 275 (2006).

[33] K.-I. Goh, G. Salvi, B. Kahng, and D. Kim, Phys. Rev. Lett. 96, 018701 (2006).

[34] J. S. Kim, K.-I. Goh, G. Salvi, E. Oh, B. Kahng, and D. Kim, Phys. Rev. E 75, 016110 (2007).

[35] F. Kawasaki and K. Yakubo, Phys. Rev. E 82, 036113 (2010).

[36] S. Furuya and K. Yakubo, Phys. Rev. E 84, 036118 (2011).

[37] Y. Sun and Y. Zhao, Phys. Rev. E 89, 042809 (2014).

[38] C. Song, L. K. Gallos, S. Havlin, and H. A. Makse, J. Stat. Mech. (2007) P03006.

[39] R. Cohen and S. Havlin, Physica A 336, 6 (2004).

[40] A. A. Moreira, J. S. Andrade, Jr., H. J. Herrmann, and J. O. Indekeu, Phys. Rev. Lett. 102, 018701 (2009).

[41] K.-I. Goh, B. Kahng, and D. Kim, Phys. Rev. Lett. 87, 278701 (2001).

[42] M. Barthélemy, Eur. Phys. J. B 38, 163 (2004). 